
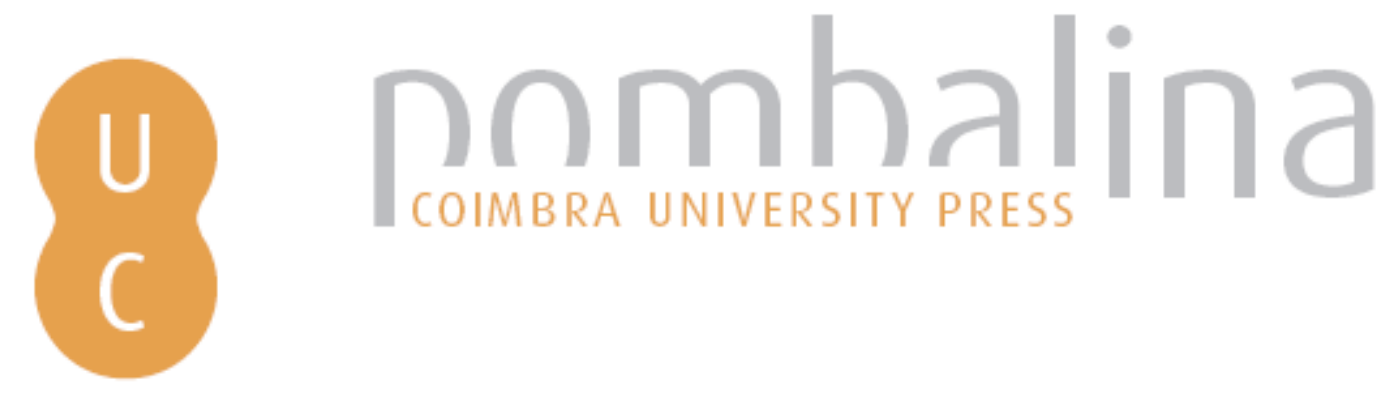

\title{
Memoria y prospectiva de la teoría de la educación
}

Autor(es): $\quad$ Gil Cantero, Fernando; Jover Olmeda, Gonzalo

Publicado por: Imprensa da Universidade de Coimbra

URL

persistente:

URI:http://hdl.handle.net/10316.2/38386

DOI:

DOI:http://dx.doi.org/10.14195/978-989-26-0486-2_8

Accessed : $\quad$ 26-Apr-2023 12:45:02

A navegação consulta e descarregamento dos títulos inseridos nas Bibliotecas Digitais UC Digitalis, UC Pombalina e UC Impactum, pressupõem a aceitação plena e sem reservas dos Termos e Condições de Uso destas Bibliotecas Digitais, disponíveis em https://digitalis.uc.pt/pt-pt/termos.

Conforme exposto nos referidos Termos e Condições de Uso, o descarregamento de títulos de acesso restrito requer uma licença válida de autorização devendo o utilizador aceder ao(s) documento(s) a partir de um endereço de IP da instituição detentora da supramencionada licença.

Ao utilizador é apenas permitido o descarregamento para uso pessoal, pelo que o emprego do(s) título(s) descarregado(s) para outro fim, designadamente comercial, carece de autorização do respetivo autor ou editor da obra.

Na medida em que todas as obras da UC Digitalis se encontram protegidas pelo Código do Direito de Autor e Direitos Conexos e demais legislação aplicável, toda a cópia, parcial ou total, deste documento, nos casos em que é legalmente admitida, deverá conter ou fazer-se acompanhar por este aviso.

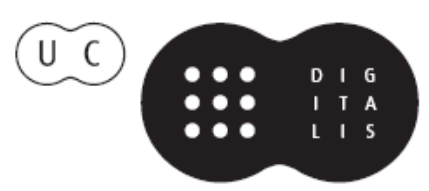


João Boavida

Ángel García del Dujo

Coordenação

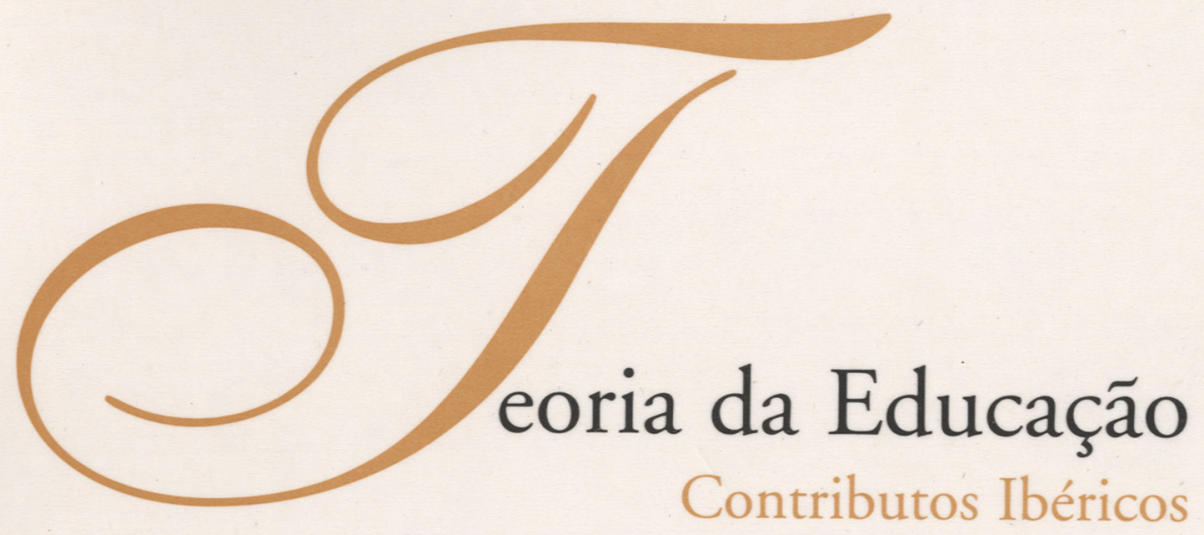


Fernando Gil Cantero

Gonzalo Jo ver OImeda

Universidad Complutense de Madrid

Memoria y Prospectiva de la Teoría de Ia Educación

\section{Configuración institucional de la Teoría de la Educación: de la especulación a la práctica}

En el año 2004 se cumplió el primer centenario de la provisión de la Cátedra de Pedagogía Superior en el doctorado de la Facultad de Filosofía y Letras de la Universidad de Madrid, precedente de la institucionalización universitaria de los estudios de pedagogía en España. La evolución del conocimiento pedagógico a lo largo de estos cien años puede considerarse la historia de una progresiva diversificación, cercana, en algún momento, a la disolución. Por poner sólo un ejemplo, los ocho cursos de enseñanzas pedagógicas propuestos en el año 1932-33 en la entonces llamada Universidad de Madrid (Universidad de Madrid, 1932, pp. 37 y 38) se han convertido en más de ochenta asignaturas ofertadas por la misma universidad en el curso 2004-2005 sólo para la Licenciatura en Pedagogía, entre troncales, obligatorias, optativas y libre configuración, anuales o cuatrimestrales. Puede dudarse que esta proliferación se corresponda con un avance paralelo del conocimiento acerca de la educación, pero plantear así el fenómeno significa pasar por alto que las disciplinas no son sólo parcelas de conocimiento que respondan a aspectos delimitables de la realidad, sino, fundamentalmente, construcciones sociales e históricas, que se desarrollan mediante tensiones, solapamientos, contraposiciones... en el seno de comunidades académicas (Foucault, 1991 
pp. 47-74, Geertz, 1994, pp. 31-49). El cuadro siguiente plasma, de manera sintética, los resultados de un rastreo a través del Boletín Oficial del Estado sobre los orígenes y evolución legislativa de la Teoría de la Educación en los planes de estudios de Pedagogía o Ciencias de la Educación de las 23 universidades españolas que ofertan Licenciatura en Pedagogía(1).

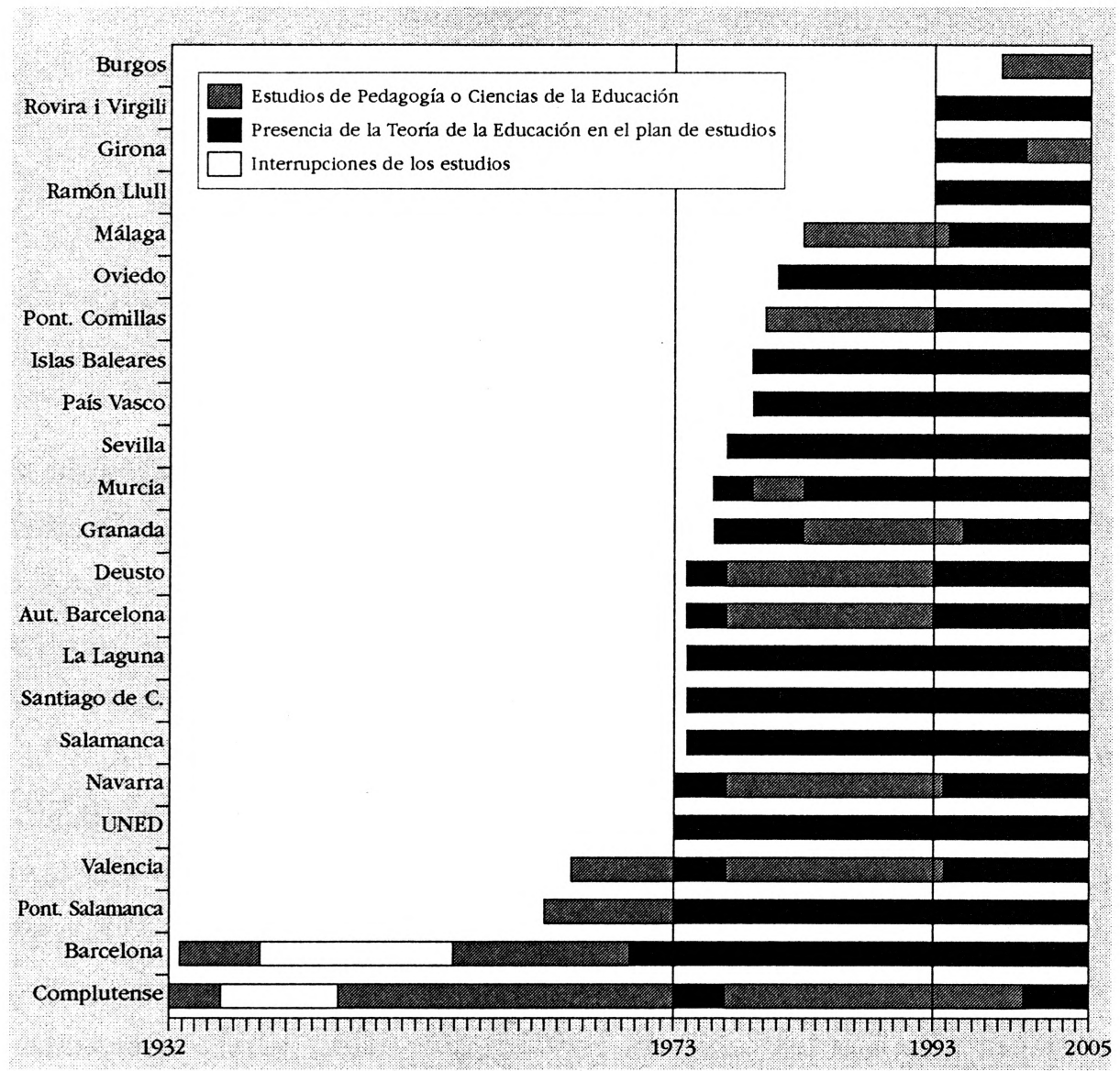

Como muestra el gráfico, en el proceso de configuración institucional de la disciplina como materia de enseñanza, pueden distinguirse claramente tres etapas.

(1) ei gráfico y los cuadros posteriores tienen sólo en cuenta la evolución legislativa de los planes de estudio aprobados, no las contingencias de sus aplicaciones. Así, en la Universidad Pontificia Comillas se oferta actualmente sólo el segundo ciclo del plan del año $2000 \mathrm{y}$, por tanto, no se imparte la asignatura de Teoría de la Educación, que dicho plan incluye en el primer curso. 


\subsection{Los antecedentes: $1932-1973$}

La primera etapa de esta configuración comprende desde la creación de las primeras Secciones de Pedagogía en las Universidades de Madrid y Barcelona, a comienzos de los años treinta, hasta el inicio de los setenta. Durante estos cuarenta años, la materia está ausente en las pocas universidades que disponen en esta época de estudios de pedagogía(2). El campo de conocimiento que se mueve en el entorno de la Teoría de la Educación es asumido por las disciplinas pedagógicas clásicas: Pedagogía, Pedagogía General y Filosofía de la Educación. La primera referencia a la Teoría de la Educación la encontramos en el llamado Plan Maluquer de la Universidad de Barcelona, de 1969. La asignatura dependía del Departamento de Ciencias Teoréticas e Historia de la Educación, creado en 1966 (Borrel, Benedito y Millán, 1979, pp. 29-34 y 50). Iniciando una tendencia que se consolidará en los años posteriores, surge ya aquí con el carácter de una asignatura especializada (asignatura específica de licenciatura) que sigue a otras materias de tipo más introductorio (en este caso, Introducción a la Pedagogía) y paralela a otras asignaturas del plan de estudios de las que, por tanto, se diferencia (en este caso, Filosofía de la Educación).

\subsection{La búsqueda de identidad: 1973-1993}

El segundo periodo comprende desde 1973, fecha de aprobación del llamado Plan Suárez, hasta 1993, año en el que empiezan a aprobarse los primeros planes de estudio adaptados al decreto de directrices generales

(2) No obstante, es posible señalar un precedente remoto indirecto en el catálogo de ensenanzas ofertadas por la Sección de Pedagogía de la Facultad de Filosofía y Letras de la Universidad de Madrid en el año 1935-36. Ese año la profesora María de Maeztu incluye dentro de su curso de Didáctica especial y problemas actuales de la educación un bloque trimestral de contenido sobre «Teoría de la educación. La pedagogía científica. Técnica y experimentación» (Universidad de Madrid, 1935, p. 59). 
de la Licenciatura en Pedagogía de 1992. En este periodo se produce una considerable expansión de los estudios de Pedagogía (Ciencias de la Educación, en la denominación de la época) en las universidades españolas, movido por el propio proceso de desarrollo universitario. En menos de 10 años, de L973 a 1981, el número de universidades que ofertan estos estudios en nuestro país se multiplica casi por cinco, pasando de cuatro a diecinueve. Es el periodo de los cambios políticos y legislativos fundamentales que afectan al sistema social y al sistema educativo de nuestro país. Ya en el inicio del periodo, la Ley General de Educación de 1970 supondrá la cristalización de las transformaciones sociales de los años sesenta, y tendrá repercusiones directas en nuestra disciplina. En primer lugar, por el intento de desarrollo y modernización del sistema de educación, lo que supuestamente exigiría una preparación acorde de los profesionales tanto de la enseñanza en sus diferentes niveles como de quienes desempeñan otras funciones pedagógicas(3). En segundo lugar, la Ley de 1970 concede cierta autonomía a las universidades, en materia de investigación y docencia, y flexibiliza los planes de estudio, que estructura en tres ciclos. Ambas circunstancias se concretan en la aprobación de unos nuevos planes para el primer ciclo de las Facultades y Divisiones de Filosofía y Ciencias de la Educación, en los que se generaliza la inclusión de la Teoría de la Educación, tanto en las universidades que ya contaban con estudios de Ciencias de la Educación como en las que se van implantando posteriormente.

Según esa tendencia que ya habíamos detectado unos años antes en la Universidad de Barcelona, en estos nuevos planes la Teoría de la Educación no se entiende como una asignatura de iniciación (papel que la nueva regulación asigna a la asignatura, común con otros estudios de Filosofía y Ciencias de la Educación, de Introducción Empírica a las Ciencias de la

(3)Como ha señalado Escolano, estas expectativas no se verían, sin embargo, satisfechas, debido a algunas medidas contradictorias de la Ley General de Educación que si, por un lado, parecía fomentar el desarrollo de la pedagogía, por otro le restaba, de hecho, presencia en el mundo escolar. Las reformas posteriores no han logrado superar los efectos de esta contradicción (Escolano, 2002, p. 202). 
Educación), sino con el carácter de un conocimiento especializado que el plan reserva para los alumnos de Ciencias de la Educación, normalmente situada en el segundo 0 tercer año de la carrera.

A partir de este momento inicial, la Teoría de la Educación correrá diferente suerte en las distintas universidades del país. En algunas de ellas se consolida definitivamente, mientras que en otras vuelve a ser pronto reemplazada por materias con denominaciones de los planes anteriores. En estos años, la Teoría de la Educación se ve así afectada por la búsqueda de identidad epistemológica en relación —en ocasiones, pugna - con otras disciplinas de su entorno. La configuración de los planes de estos años revela esta ambigüedad, de modo que todavía en 1988 podía afirmarse en el marco del II Congreso Nacional de Teoría de la Educación, que «en España, el concepto de Teoría de la Educación es tan reciente como polivalente» (Ibáñez-Martín, 1989, p. 144). En algunos de estos planes, la Teoría de la Educación parece entenderse como alternativa a la antigua Pedagogía General, a la que sustituye. En otros, en cambio, esta situación no es tan clara. En algunos, la inclusión de la Teoría de la Educación no impide la presencia en el mismo plan de estudios de Pedagogía General o Pedagogía Sistemática. Por último, en ciertos casos, como en el plan de la Universidad Nacional de Educación a Distancia de 1973 o en el de la Universidad de Valencia de 1978, se asimila en su denominación a la Filosofía de la Educación(4). En la Universidad Complutense, sin añadir tal matiz en el nombre, su docencia estuvo de hecho a cargo de los profesores de esta disciplina, lo mismo que durante algún tiempo sucedió en la Universidad Autónoma de Barcelona con la de Teorías de la Educación.

Como pauta general, a lo largo de este periodo las universidades que incluyen junto con Teoría de la Educación la asignatura de Filosofía de la Educación, omiten en el mismo plan de estudios la de Pedagogía General. En

(4) Con los nombres de Teoría de la Educación (Filosofía de la Educación), en la primera, y Filosofía de la Educación (Teoría de la Educación), en la segunda. 
estas universidades cabe, por tanto, suponer que se produce una vinculación de la Teoría de la Educación como alternativa a esta última asignatura. Por el contrario, las universidades que incluyen junto con nuestra disciplina las de Pedagogía General o Pedagogía Sistemática suelen omitir en el mismo plan de estudios la Filosofía de la Educación. Cabe suponer, por tanto, que aquí la vinculación es con respecto a esta otra asignatura. Si estos supuestos son correctos, durante los años setenta se habría dado inicialmente una adscripción más fuerte de la Teoría de la Educación con la Filosofía de la Educación, para ir adquiriendo hacia el final de esa década y el comienzo de la siguiente el sentido contrario(5).

Presencia conjunta de Teoria de la Educación, Pedagogía General o Sistemática y Filosofia de la Educación en los Planes de Estudio (1973-1993)(6)

\begin{tabular}{ccccc}
\hline UNIVERSIDAD & PLAN & $\begin{array}{c}\text { Pedag. General } \\
\text { o Sistemática }\end{array}$ & $\begin{array}{c}\text { Teoría de la } \\
\text { Educación }\end{array}$ & $\begin{array}{c}\text { Filosofía de la } \\
\text { Educación }\end{array}$ \\
\hline \hline Valencia & $73 / 76$ & $\mathrm{X}$ & $\mathrm{X}$ & \\
P. Salamanca & $73 / 77$ & $\mathrm{X}$ & $\mathrm{X}$ & \\
La Laguna & $74 / 76$ & $\mathrm{X}$ & $\mathrm{X}$ & $\mathrm{X}$ \\
Barcelona & $75 / 76$ & & $\mathrm{X}$ & \\
Murcia & 78 & $\mathrm{X}$ & $\mathrm{X}$ & \\
Sevilla & 79 & $\mathrm{X}$ & $\mathrm{X}$ & $\mathrm{X}$ \\
La Laguna & 79 & & $\mathrm{X}$ & $\mathrm{X}$ \\
UNED & $79 / 82$ & & $\mathrm{X}$ & $\mathrm{X}$ \\
Santiago de Comp. & $79 / 82$ & $\mathrm{X}$ & $\mathrm{X}$ & \\
Murcia & 83 & & $\mathrm{X}$ &
\end{tabular}

(5) Como muestra de esta ambivalencia, muchos de los tratados de Teoría de la Educación de estos años, desde un perfil más cercano a la Filosofía de la Educación, prestan especial atención a las relaciones de aquélla con ésta, para resaltar tanto sus semejanzas como sus diferencias (Campillo, 1974, pp. 17-22; Fermoso, 1976, pp. 103-113; Capitán, 1977, pp. 51-53).

(6) se recogen sólo aquellos planes que incluyen, junto con Teoría de la Educación, alguna de las otras dos disciplinas. Vease también la nota 1. 
La Ley de Reforma Universitaria, de 1983, quiso reforzar la organización departamental de las universidades y supuso una nueva estructuración por áreas de conocimiento. Como consecuencia, se originó el Área de Conocimiento de Teoría e Historia de la Educación (con un precedente cercano en el Plan de Estudios de 1977 de la Universidad Autónoma de Barcelona(7), y más remoto en el Departamento de Ciencias Teoréticas e Historia de la Educación de la Universidad de Barcelona) a la que se adscribió la docencia de la asignatura.

A lo largo de los años ochenta se va imponiendo en el círculo académico de la disciplina su consideración como una forma de conocimiento científico y tecnológico, remitiendo a un segundo plano los planteamientos de carácter más axiológico propios de la Filosofía de la Educación(8). Como explicaría años más tarde Escámez, este proceso de autodefinición de la Teoría de la Educación estuvo animado por el propósito de dotar de mayor consistencia y cientificidad al conocimiento de la educación y el trabajo de los educadores, bajo la presunción de que la educación es una actividad técnicamente regulable (Escámez, 1988). Esta evolución es impulsada por tres factores. En primer lugar, la organización, en los años setenta, del Seminario de Pedagogía Cibernética y Teoría General de Sistemas en el Departamento de Pedagogía Sistemática de la Universidad de Barcelona, dirigido por el profesor Alejandro Sanvisens, que constituirá uno de los principales focos para el desarrollo del enfoque tecnológico de la Teoría de la Educación. En

(7) Donde se establece una programación por áreas en el segundo ciclo, introduciendo la denominación de Area de Teoría e Historia de la Educación.

(8) Esto no significa que desde la Teoría de la Educación de cuño científico-tecnológico no se tuviese también en cuenta que la educación tiene un lado ético, ni que desde la Filosofía de la Educación de orientación normativo-axiológica no se viese la necesidad de cierta regulación técnica de los procesos educativos. La cuestión no es de dimensiones, sino de posiciones, acerca de la educación y su conocimiento. Ya en varios trabajos publicados desde inicios de los noventa hemos insistido en esta diferencia (Bárcena, Gil y Jover, 1991 y 1993; Gil, 1997, Jover, 2003). Hoy los límites entre estas dos perspectivas están más desdibujados, como hemos tratado de mostrar en un estudio anterior sobre la evolución en nuestro país del campo de conocimiento de la Filosofía de la Educación (Jover, 2001). Y lo están porque de ser enfoques dominantes, han pasado a constituirse en perspectivas que forman, junto a otras y al mismo nivel, un entramado de accesos diversos a la complejidad de la educación que a menudo presentan zonas de solapamiento. 
segundo lugar, el acceso a las categorías superiores de la carrera docente, a partir de la segunda mitad de los setenta, de nuevos profesores, «hijos de la postguerra» (Colom, 2003, p. 156), que intentan implantar un estilo nuevo en la pedagogía. Por último, el inicio, en 1982, del actual Seminario Interuniversitário de Teoría de la Educación, que pronto se erige en portavoz de dicho enfoque(9).

\subsection{La consolidación: 1993-actualidad}

La modificación de los planes de estudio de pedagogía, a que dieron lugar las normativas emanadas de la Ley de Reforma Universitaria, supuso la consolidación de la Teoría de la Educación como materia de enseñanza a comienzos de los noventa. Puede situarse en 1993, año en el que comienzan a aprobarse los nuevos planes adaptados a la normativa nacida de la LRU, el momento de afianzamiento definitivo de la disciplina, incluida como materia troncal en el Decreto 915/1992, de 17 de julio, que establece las directrices generales de los planes de estudio de Licenciatura en Pedagogía. En los nuevos planes, la asignatura suele incluirse en el primer curso de estudios, dotándola quizás de ese carácter de introducción que era menos evidente en las épocas anteriores.

(9) Dando cuenta de los antecedentes y circunstancias que rodearon esta iniciativa, recordaba Marín Ibáñez, uno de los participantes en la primera reunión, cómo, con la reimplantación de los estudios en Madrid y en Barcelona en los años cuarenta y cincuenta, la Pedagogía General (o Racional, 0 Fundamental) se configura con el sentido de una reflexión filosofica, a cargo de catedráticos como Juan Zaragüeta y Anselmo Romero, en Madrid, y Juan Tusquets, en Barcelona, si bien este último le imprime un nuevo giro con el desarrollo de la Pedagogía Comparada. A partir de los setenta, la multiplicación de universidades que ofertan estudios de Ciencias de la Educación, con la consiguiente dotación de cátedras y adjuntías, a cargo de un nuevo contingente de profesores, algunos de ellos procedentes de la materia de Introducción a las Ciencias de la Educación, contribuye a dar un perfil variopinto a la disciplina, que según los casos, los intereses, la formación previa, y las líneas de trabajo se orienta preferentemente desde la Sociología, la Antropología, la Filosofía, la Tecnología, la Teoría de Sistemas, etc. Es entonces cuando, en una reunión de catedráticos y agregados de pedagogía, hay quienes proponen el abandono de la Pedagogía General y su sustitucion por la Teoría de la Educación, aunque ya entonces se ven aflorar las tensiones en la demarcación de esta nueva disciplina (Marín Ibáñez, 1983). 
Si realizamos el mismo examen comparativo que en el periodo anterior sobre la inclusión de disciplinas conexas, el panorama que obtenemos es ahora bastante diferente. Sólo una universidad dispone de la asignatura de Pedagogía General además de Teoría de la Educación, mientras que muchas de ellas incluyen, junto a ésta, Filosofía de la Educación como materia obligatoria u optativa(10).

Presencia conjunta de Teoria de la Educación, Pedagogía General o Sistemática y

Filosofia de la Educación en los Planes de Estudio (1993-actualidad)(11)

\begin{tabular}{|c|c|c|c|c|}
\hline UNIVERSIDAD & PLAN & $\begin{array}{l}\text { Pedagogía } \\
\text { General }\end{array}$ & $\begin{array}{c}\text { Teoria de la } \\
\text { Educación }\end{array}$ & $\begin{array}{l}\text { Filosofía de la } \\
\text { Educación }\end{array}$ \\
\hline Aut. Barcelona & 93 & & $\mathrm{x}$ & $\mathrm{x}$ \\
\hline P. Comillas & 93 & & $\mathrm{x}$ & $\mathrm{x}$ \\
\hline Oviedo & 93 & & $\mathrm{x}$ & $\mathrm{x}$ \\
\hline Girona & 93 & & $\mathrm{x}$ & $\mathbf{x}$ \\
\hline Valencia & 94 & & $\mathrm{x}$ & $\mathrm{x}$ \\
\hline Navarra & 94 & & $\mathrm{x}$ & $\mathrm{x}$ \\
\hline La Laguna & 94 & & $\mathrm{x}$ & $\mathrm{x}$ \\
\hline Málaga & 94 & & $\mathrm{x}$ & $\mathrm{x}$ \\
\hline UNED & 95 & & $\mathrm{x}$ & $\mathrm{x}$ \\
\hline Granada & 95 & & $\mathrm{x}$ & $\mathrm{x}$ \\
\hline Santiago de C. & 96 & & $\mathrm{x}$ & $\mathrm{x}$ \\
\hline Ramón Llull & 96 & $\mathrm{x}$ & $\mathrm{x}$ & $\mathrm{x}$ \\
\hline Islas Baleares & 97 & & $\mathrm{x}$ & $\mathrm{x}$ \\
\hline Sevilla & 98 & & $\mathbf{x}$ & $\mathrm{x}$ \\
\hline UNED & 99 & & $\mathrm{x}$ & $\mathrm{x}$ \\
\hline País Vasco & 99 & & $\mathrm{x}$ & $\mathrm{x}$ \\
\hline Málaga & 99 & & $\mathrm{x}$ & $\mathrm{x}$ \\
\hline Complutense & 00 & & $\mathrm{x}$ & $\mathrm{x}$ \\
\hline Barcelona & 00 & & $\mathrm{x}$ & $\mathrm{x}$ \\
\hline valencia & 00 & & $\mathrm{x}$ & $\mathrm{x}$ \\
\hline P. Comillas & 00 & & $\mathrm{x}$ & $\mathbf{x}$ \\
\hline Oviedo & 00 & & $\mathrm{x}$ & $\mathrm{x}$ \\
\hline Ramón Llull & 00 & $\mathrm{x}$ & $\mathrm{x}$ & $\mathrm{x}$ \\
\hline Granada & 01 & & $\mathrm{x}$ & $\mathrm{x}$ \\
\hline Santiago de Comp. & 01 & & $\mathrm{x}$ & $\mathrm{x}$ \\
\hline
\end{tabular}

(10) La única excepción a esta situación general la representa la Universidad Ramón Llull, en la que, tanto en el Plan de 1993 como en la última adaptación del 2000, conviven Teoría de la Educación, Pedagogía General y Filosofía de la Educación.

(11) véase nota 6. 
Frente a la indefinición del periodo anterior, a partir de su inclusión como materia troncal, la Teoría de la Educación parece proponerse indiscutiblemente como sustituía de la antigua Pedagogía General. Pero no se trata simplemente de un cambio de denominación, sino que tras él se encuentra la pretensión de dotar de una orientación diferente al conocimiento de la educación. Así, para Colom da teoría de la educación no suple a la pedagogía general 0 racional. Esta última es de carácter especulativo, mientras que la teoría de la educación es fundamentalmente utilitarista y pragmatista. Su objetivo se centra en mejorar la práctica educativa a través del conocimiento que le propician las ciencias de la educación con sus diversas metodologías» (Colom, 1997, p. 154).

Se asume en nuestro entorno que este intento de modernización que lleva de la Pedagogía General a la Teoría de la Educación supuso también un cambio de referentes, de la pedagogía de tradición germana, de orden más filosófico y especulativo, a la Educational Theory anglosajona, de carácter más positivista y práctico (Colom, 1992, pp. 12-14)(12) Ahora bien, la recepción del flujo internacional de ideas y paradigmas epistemológicos no se produce en un estado puro, sino que suele quedar matizada por las ideosincrasias locales, las tradiciones preexistentes, los esquemas de interpretación predominantes, etc.(13) Por este motivo, no hay que extrañarse de que, mientras en nuestro país se miraba al mundo anglosajón en busca de una Teoría de la Educación más práctica como alternativa a la antigua pedagogía, en el Reino Unido hubiese quienes se lamentaban de la falta de una pedagogía similar a la que tradicionalmente se ha cultivado en el continente, y de que en su lugar «nuestro planteamiento de la teoría y la

(12) Concretamente, existe coincidencia en señalar la publicación en castellano, en 1980, de la Introducción a la teoría de la educación de T.W. Moore (1983, 2a ed.), publicada originalmente en 1974, como momento clave en esta evolución (Colom, 2003, p. 125; Sarramona, 2003, p. 164).

(13) En lo que se refiere a la difusión del conocimiento pedagógico, hay que destacar en esta línea los trabajos del comparativista Jürgen Schriewer y su grupo de investigación en el Instituí für Allgemeine Pddagogik de la Universidad Humboldt de Berlín (Schriewer, 1996 y 2000). 
práctica educativa haya tendido a ser de carácter poco especializado y profundamente pragmático» (Simón, 1999, p. 34)(14)

Para apreciar el sentido de esta observación, hay que recordar, aunque sea rápidamente, que la Teoría de la Educación británica, que se acoge en nuestro país como referente, sufrió durante la segunda mitad del siglo inmediatamente pasado diversos vaivenes. En 1942, Charles D. Hardie se quejaba de que «el presente estado de la teoría de la educación, con sus numerosas doctrinas en conflicto, no puede considerarse en absoluto satisfactorio» y abogaba, en consecuencia, por una renovación similar a la operada en el campo de la filosofía por Moore, Broad y Wittgenstein (Hardie, 1968, p. XIX)(15) a partir de la segunda mitad de los sesenta, el estudio teórico de la educación experimentó uno de sus mayores impulsos, gracias a iniciativas como las emprendidas por el filósofo de la educación Richard Stanley Peters en el Instituto de Educación de la Universidad de Londres, y favorecido por ciertas medidas oficiales, como el informe Robbins, que sugería el desarrollo de las titulaciones específicas de estudios sobre educación y que la formación no se enfocase exclusivamente hacia la capacitación profesional. Pero sólo una década más tarde se producirá un giro distinto. Diversos autores lamentarán que un excesivo entusiasmo por la teoría hubiese llevado a un descuido de la práctica, y pedirán una mayor vinculación con la misma, iniciando una orientación que se reforzará en los ochenta, con el surgimiento de campos híbridos, como los estudios curriculares y de administración, muy centrados directamente en la práctica. A mediados de esa década, Dearden constataba la situación generada señalando que en los estudios sobre educación en el Reino Unido «no hay nada que pueda ser visto como una ciencia unificada de la educación» (Dearden, 1984, p. 38). La consecuencia institucional de este giro, señala a su vez Hirst, fue la progresiva marginación, a partir de la segunda mitad de los ochenta, de las disciplinas académicas clásicas sobre el estudio de la educación (Hirst, 1998, p. 21).

(14) Reedición de un trabajo originalmente publicado a comienzos de los años ochenta.

(15) La referencia procede de la tercera reimpresión de la edición americana de esta obra, publicada originalmente en el Reino Unido en 1942 por Cambridge University Press. 
Mientras tanto, en nuestro país, desde unos antecedentes distintos, la Teoría de la Educación aspira a superar el riesgo de disolución de la pedagogía bajo el modelo de las ciencias de la educación, entonces aún imperante, y a convertirse en cierto modo en ese conocimiento científico sintetizador que Dearden ya no puede encontrar por ningún lado en el suyo. La diferencia de ritmos entre ambos contextos, induce a pensar que, más que copiar soluciones que en el propio Reino Unido venían ya siendo objeto de un amplio debate(16), tras la pretensión de convertir la Teoría de la Educación en un conocimiento científicamente fundado y tecnológicamente orientado, latía en nuestro caso una voluntad de modernización animada por una motivación más circunstancial, o política, que epistemológica, hasta donde ambas cosas sean separables(17). Se trataba en definitiva de hacer patente la ruptura con la tradición anterior (Sarramona, 2003, p. 165), 0, como también se ha dicho, de distanciarse de «los caminos que marcara la pedagogía de la lucha ascética» (Colom, 2003, p. 124).

2. La investigación en Teoría de la Educación: hacia los múltiples discursos

En un balance de la evolución experimentada por la Teoría de la Educación durante las dos últimas décadas del siglo inmediatamente pasado, se lamentaba hace unos años Sarramona de que «el cambio de denominación

(16) Tal como se manifestó, por ejemplo, en la conocida discusión entre D. J. O'Connor y P. H. Hirst, iniciada en 1957 con la publicación del libro de 0'Connor Introduction to the Philosophy of Education, (0'Connor, 1967, 60 reimp.) y prolongada hasta comienzos de los ochenta con la publicación a cargo de Hirst del trabajo de revisión incluido en su libro Educational Theory and its Foundation Disciplines (Hirst, 1983).

D7) colom y Rincón han planteado recientemente la misma idea: «La gran dificultad epistemológica de la Pedagogía es consecuencia de su herencia histórica, lo que equivale a decir que la problemática conceptual de la educación, más que epistemológica, es histórica» (Colom y Rincón, 2004, pp. 39-40). 
de 'Pedagogía' por 'Teoría de la educación' no ha resuelto las expectativas de quienes la promovieron, porque el deseo de buscar una fundamentación más empírica y no exclusivamente filosófica a la actuación educativa, que se reservaría para la Filosofía de la educación, se ha visto condicionada por la dinámica corporativista de los docentes universitarios, que ha llevado a calificar a todas las materias abarcadas en el Área de Teoría e Historia de la Educación como de meramente especulativas, sin favorecer con ello la vinculación de la teoría con la práctica» (Sarramona, 2000, p. 8)(18).

Sin negar el peso de los motivos corporativistas, que todos hemos conocido, y muchos padecido, cabe ensayar algunas interpretaciones complementarias de lo que ha sucedido. En el año 2002, uno de los autores de este trabajo realizó un pequeño análisis de la evolución de la investigación en Teoría de la Educación en nuestro país(19), tomando como referencia un estudio publicado diez años antes por Martínez y Buxarrais (Martínez Martín y Buxarrais, 1992). A partir de una encuesta realizada entre los especialistas del ámbito, estos autores identificaron los campos propios de la investigación teórica en educación, distinguiendo un bloque de investigación de carácter básico y otro de carácter aplicado. El primer bloque se refiere a las áreas de estudio e investigación fundamentalmente, aunque no exclusivamente, de tipo descriptivo, explicativo, comprensivo o reflexivo y discursivo, e incluye siete campos o áreas temáticas: 1) metodológica y 18191819

(18) ei recurso tan recurrente a poner como ejemplo de saber teórico puro a la Filosofía de la Educación convendría matizarlo, aunque sólo sea porque presupone una uniformidad de criterio que no existe ni en la Teoría de la Educación ni en la Filosofía de la Educación. Basta, para comprobarlo, echar una mirada a la ya larga tradición, dentro y fuera de nuestro país, de una Filosofía práctica de la Educación, bien distinta, por cierto, de la que proponía T. W. Moore. Lo que diferencia a la Teoría de la Educación y a la Filosofía de la Educación no es que ésta estudie la «educación como objeto de conocimiento con independencia de su puesta en prácticas Todo saber déla educación debe referirse, en algún momento, a la práctica. Lo que las diferencia es que la primera se ocupa de la práctica de un modo prescriptivo o normativo inmediato dirigido a las condiciones estructurales de las acciones educativas para que sean más eficaces, mientras que la segunda se ocupa, entre otras cuestiones, de la práctica de un modo prescriptivo 0 normativo mediato dirigido a las condiciones de sentido humanizador de las finalidades de las acciones educativas (Jover, 2001; Gil, 2003).

(19) Este análisis se elaboró para el proyecto docente presentado en el concurso oposición a la Cátedra de Teoría de la Educación de la Universidad Complutense de Madrid, celebrado en octubre de 2002 (Jover, 2002). 
conceptual; 2) antropológica; 3) psicológica; 4) sociológica; 5) filosófica y axiológica; 6) contextual y 7) política. El segundo bloque agrupa los temas de investigación más directamente enfocados a la acción pedagógica en los que trabajan los especialistas del ámbito en cinco áreas temáticas: 8) animación sociocultural; 9) educación de adultos; 10) educación moral; 11) pedagogía laboral y 12) profesiones educativas. Como advierten los autores de la revisión, la delimitación de estos dos bloques no es estricta y en la práctica de la investigación pedagógica ambos se entrecruzan, haciendo su separación a menudo difícil, «por lo que debe entenderse como referencia que sólo queda confirmada en función de los indicadores que cada investigador atribuya a las áreas y subáreas de estudio que manifieste como preferentes en su trabajo concreto»(ibid., p. 25).

Partiendo de esta clasificación de Martínez y Buxarrais, se rastreó la evolución de la investigación a lo largo de los últimos veinte años, por medio de los trabajos en publicaciones periódicas de los especialistas españoles recogidas en la base de datos ISOC (Ciencias Sociales y Humanas) del Consejo Superior de Investigaciones Científicas $(20)$. Con efectos analíticos, se optó por mantener la misma clasificación general propuesta a partir del trabajo desarrollado por Martínez y Buxarrais a comienzos de los noventa, si bien la evolución durante los años transcurridos obliga a realizar algunas matizaciones mediante el siguiente cuadro de descriptores, que en algunos casos completan, introduciendo nuevas líneas de trabajo, la descripción realizada por estos autores en $1992:{ }^{* 10} * 10$

(20)Con una finalidad operativa, se consideraron especialistas del ámbito a quienes, a lo largo de esos veinte años, habían participado por lo menos tres veces en los Seminarios Interuniversitários de Teoría de la Educación, que, como apuntamos antes, vienen cejebrándose ininterrumpidamente cada año desde 1982, con el objetivo, precisamente, de fomentar, trazar las líneas y poner en común la investigación que se desarrolla en el ámbito. Este criterio dio lugar a la identificación de 79 autores, cuyos trabajos se consultaron. La consulta a la base de datos se realizó en los meses de abril y mayo de 2002, empleando la última versión disponible en ese momento. Una vez suprimidas las aportaciones de carácter más divulgativo, se obtuvieron 743 referencias en las que se basó el análisis. 
Á reas Temáticas del estudio teórico de la educación

A) De carácter básico

1. Metodológica y conceptual: Concepto de educación, metodología de investigación, epistemología, racionalidad pedagógica, historia de las teorías e instituciones educativas, documentación pedagógica.

2. Antropológica: Antropología filosófica, antropología biológica, antropología social y cultural.

3. Psicológica: Teoría del aprendizaje, procesamiento de información, procesos cognitivos, motivación, educación especial, autoconcepto.

4. Sociológica: Pedagogía Social, aprendizaje social, desarrollo comunitario, marginación y bienestar social.

5. Filosófica y axiologica: Teoría axiológica, finalidades de la educación, dimensión ética de la educación.

6. Contextual: Instituciones educativas, conflictividad escolar, proceso instructivo, currículo, competencias, niveles de enseñanza, enseñanza a distancia, ámbitos de educación no formal e informal, medios de comunicación, sociedad de la información, pedagogía penitenciaria.

7. Políica: Organización y administración de la educación, sistema educativo, reforma educativa, estudios comparados, política educativa internacional, prospectiva.

B) De carácter aplicado

8. Animación socio-cultural.

9. Educación de adultos.

10.Educación moral: Formación de actitudes, educación moral, educación religiosa, educación intercultural, educación cívica, educación ambiental, educación para el desarrollo, educación para la salud (secundariamente otras dimensiones educables de la persona: intelectual, estética, afectiva, etc.).

11. Pedagogía laboral: Programas de formación en la empresa.

12. Profesiones educativas: Profesorado, funciones pedagógicas, deontologia profesional.

Para comprobar la evolución de la investigación en el campo de conocimiento, se distinguieron dos periodos, según un criterio puramente cronológico, de 1981 a 1990 y de 1991 a 2002, y se clasificaron los trabajos correspondientes a cada uno de ellos en función del esquema anterior. El gráfico siguiente recoge los resultados del análisis, en términos porcentuales, en cada una de las doce áreas temáticas para los dos periodos considerados $\left({ }^{21}\right)$.

(21) Los porcentajes indican el peso relativo de cada área en el periodo correspondiente. Su suma dentro de cada periodo es superior a 100, ya que muchos de los trabajos pueden incluirse en más de una area. En casos excepcionales, hay trabajos que se han situado hasta en tres áreas, como máximo. 


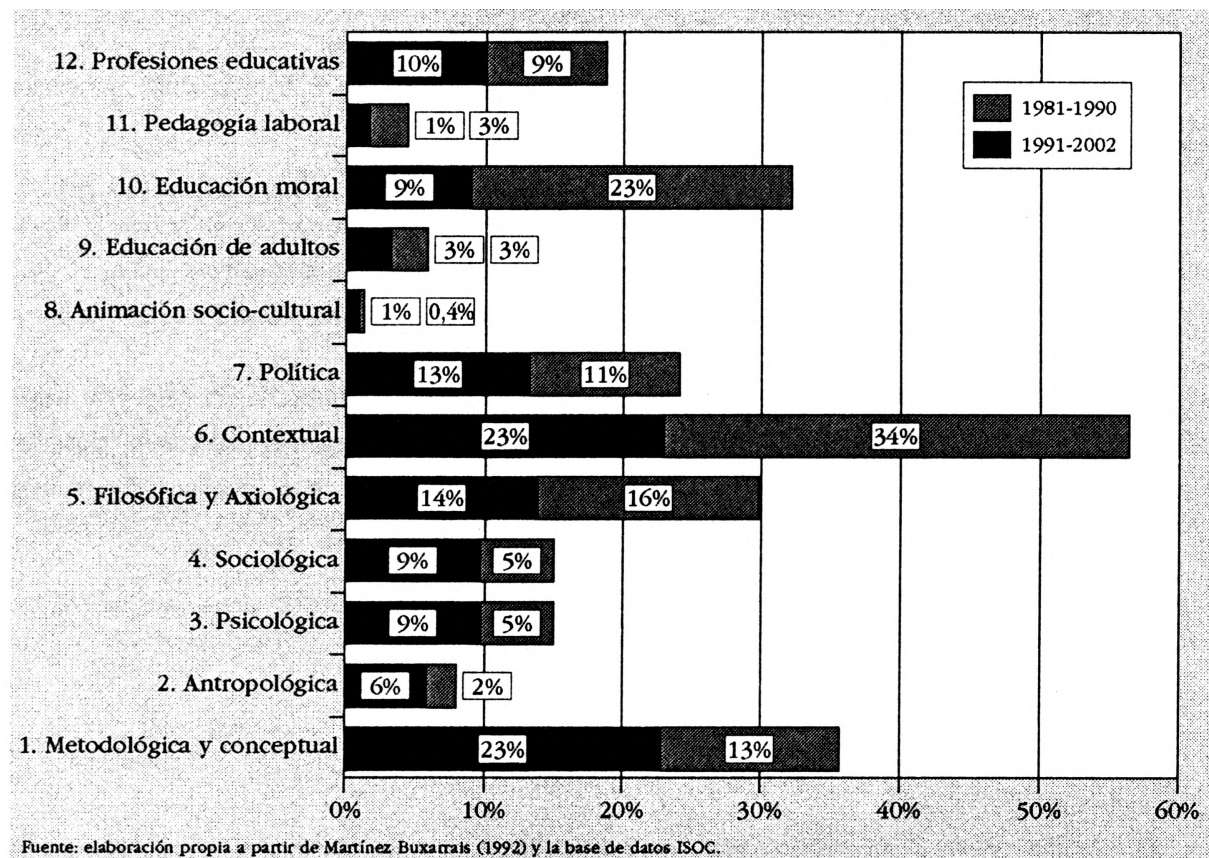

Evolución de las áreas temáticas de investigación en Teoria de la Educación.

Este análisis permite extraer algunas conclusiones interesantes sobre el sentido que ha ido adquiriendo la Teoría de la Educación. Por un lado, observamos que en el bloque de carácter básico disminuye considerablemente el peso relativo de las áreas metodológica-conceptual, antropológica, psicológica y sociológica, y en menor medida del área política, mientras que aumenta levemente el de las áreas filosófico-axiológica, y muy considerablemente la contextual. Ésta última incluye los estudios sobre los agentes, medios y entornos de la educación formal, no formal e informal. Su desarrollo se ha visto favorecido por el surgimiento de nuevos entornos de intervención pedagógica (pedagogía de la ciudad, pedagogía penitenciaria, sociedad de la información, etc.), que, junto con los tradicionales procesos de la educación formal, han sido objeto de considerable atención investigadora. Como señalan Martínez y Buxarrais, se trata de un área de trabajo que, si 
bien se ha incluido dentro del bloque de estudios de carácter básico, reviste una ineludible vertiente aplicada, orientada al incremento de la eficacia de la acción en estos diferentes entornos de educación, llegando incluso a presentar a veces una gran afinidad con las investigaciones didácticas (Abid, p. 26).

En lo que se refiere al bloque de investigación de carácter aplicado, observamos que el mayor incremento se ha producido en el área de educación moral, en lo que sin duda ha influido tanto el desarrollo de la línea específica de investigación sobre esta dimensión de la tarea educadora, ya muy asentada con grupos de investigación reconocidos en varias de nuestras universidades (Escámez, 2003), como su extensión a otros intereses conexos, especialmente la educación cívica y la educación intercultural(22). $Y$, si es cierto que el trabajo que se realiza en estos campos de acción pedagógica incluye también el esfuerzo de fundamentación, lo es también que otra gran parte de la investigación está realizada con una intención de aplicación más directa(23).

Pero, quizás, lo que más claramente revele en su conjunto el gráfico anterior, sea la disgregación que parece producirse de la Teoría de la Educación en múltiples discursos. Las grandes construcciones teóricas de los años ochenta, han dado lugar a enfoques mucho más contextualizados, más concretos. Ya no interesa la Teoría de la Educación como tal, sino la Teoría de la Educación del mundo infantil, de la familia, de los valores, etc. Tal disgregación se entreteje con un descentramiento del foco de atención. ${ }^{2223}$

(22) menor peso relativo a lo largo de estos veinte años de la investigación aplicada en las áreas animación sociocultural, educación de adultos y pedagogía laboral, no puede interpretarse como una falta de interés por estos temas. Muy al contrario, si en el entorno de la investigación en Teoría de la Educación su presencia es más escasa se debe al desarrollo autónomo tan importante que en nuestro país ha tenido la Pedagogía Social, que ha formado un ámbito de conocimiento con identidad propia, paralelo y en cierto modo independiente de la Teoría de la Educación. Recuérdese a este respecto que el criterio seguido para la determinación de los especialistas en Teoría áe la Educación cuyas publicaciones se analizaron, fue un criterio de tipo institucional o corporativo.

(23) Téngase en cuenta que en esta área se han incluido también, por extensión, las investigaciones sobre otras dimensiones educables de la persona (educación intelectual, afectiva, estética, etc.). 
Ambos procesos, en ocasiones paradójicos, son en realidad efectos de la misma ampliación del universo educativo y la consiguiente pérdida de contornos precisos. La Teoría de la Educación se disgrega en micro discursos porque la educación se hace más volátil, y viceversa.

En los orígenes de la institucionalización de la pedagogía, ésta se asociaba estrictamente al mundo escolar. Si embargo, desde hace tiempo, recurrimos cada vez más a una versión informal de la educación, a una visión cultural de la misma, no como variable escolar, sino como condicionante de la dinámica general de la sociedad, o como proceso de desarrollo personal autónomo de sujetos adultos.

La evolución es, en cierto sentido, lógica. Tras reconocer un concepto permanente de educación que se alarga durante toda la vida; tras jalear la necesidad universal de la pedagogía porque todo educa tras asumir como válidas y necesarias todas las demandas que se vuelcan, desde el ámbito social, sobre la escuela y los profesores; tras la paulatina exclusión de los pedagogos del ámbito escolar e incluso, por qué negarlo, su rechazo por parte de los profesores, y, sobre todo, para no alargar más la lista, tras comprobar la facilidad con la que el ambiente social y los medios de comunicación logran efectos de formación, nos hemos ido sumergiendo en los problemas culturales, sociales y existenciales.

Esta ampliación del horizonte fuerza una reorientación epistemológica, y, así, no es casualidad que García Carrasco y García del Dujo terminen el último y más reciente tomo de su Teoría de la Educación afirmando: «Una Teoría de la educación ha de proponer como meta general opciones de calidad de vida bajo el manto de la variedad de situaciones y ha de afrontar cuestiones fundamentales, frente a las cuales pierden carácter taumatúrgico todas las tecnologías y se sitúan en su justo poder y valor todas las políticas culturales» (García Carrasco y García del Dujo, 2001, p.409, cursiva añadida).

El descentramiento operado tiene un lado muy positivo. La Teoría de la Educación se enriquece con la mirada puesta en un enfoque cultural, político, social, etc. de la educación. Conviene, sin embargo, reconocer 
algunas limitaciones. Para empezar, pensamos que al igual que la salud es un problema cultural que debe compaginarse con un tratamiento profesional por especialistas que concentren la atención en su objeto de estudio, la Teoría de la Educación debe integrar el análisis cultural de la educación con propuestas que mejoren la práctica educativa en situaciones concretas. El espacio informal es apasionante pero tiene el inconveniente, en este caso, de que puede desdibujar los límites de la educación haciéndola en términos prácticos inaprensible. Dicho espacio se superpone entremezcladamente con tan variados intereses sociales, culturales, políticos, etc., que es fácil, facilísimo, terminar escogiendo vías muy interesantes, política y culturalmente apasionantes, pero marginalmente pedagógicas. La educación puede terminar confundiéndose, por ausencia de enfoques analíticos rigurosos, con cualesquiera procesos sociales. La pedagogía pretende abarcarlo todo, y al abarcarlo todo, puede perder su vinculación con un objeto específico(24).

Para evitar esta limitación, deberíamos acostumbrarnos a finalizar nuestras incursiones teóricas sobre la educación como problema cultural con un viaje de vuelta hacia los espacios formales y no formales, con una mirada puesta en los procesos y agentes concretos, etc., lo que permitiría integrar tanto la enorme mediación valorativa de la educación como variable cultural como su expresión precisa en los diferentes contextos, agentes, objetivos, etc. Ni un endocrino se puede contentar con decirle a su cliente que su sobrepeso es un problema cultural, ni un pedagogo con decirle lo mismo a un profesor cuando pide soluciones, por ejemplo, para los problemas de convivencia en el aula.

(24) como uno de nosotros ha señalado en otro lugar, «hemos perdido nuestro objeto de estudio. La pérdida de los contornos de significado de muchos aspectos de la realidad, está incidiendo en la educación en el sentido de que, sin alarmismo alguno, nos hemos alejado de la indagación acerca del significado preciso de la educación y más aún de lo que significa que una persona esté educada. La Pedagogía, como todos los saberes contingentes, ha desdibujado la firmeza de su objeto de estudio al tratar de conseguir que respondiese a todo tipo de expectativas sociales» (Gil, 2001, p. 54). 


\section{Condiciones de una Teoría de la Educación integradora}

La situación de pérdida de especificidad no es, por supuesto, exclusiva de nuestro contexto. Para Walsh, gran parte de la sensación de declive experimentada por la Teoría de la Educación británica, fue motivada por la pérdida de referencia de lo educativo en algunas de las formulaciones que adoptó el giro de los setenta y los ochenta, debido a su excesiva focalización en la práctica, en detrimento de la práctica específicamente educativa, con el consiguiente rechazo a todo lo que pudiera significar conceptuación teórica de la educación. Lo que se pierde en este proceso de disgregación es la idea de la educación como una práctica singular. $\mathrm{Y}$, con ella, se va también la posibilidad de teorizarla. «El problema a considerar — escribe Walsh —es hasta qué punto el papel preponderante dado a la práctica deliberativa pone en peligro la profundidad y amplitud de la teoría. De modo inicial puede proponerse que el riesgo es minimizado cuando no perdemos de vista que la práctica en cuestión es la educación, y tenemos cuidado de abarcar esta práctica en su extensión total» (Walsh, 1993, p. 43).

En nuestro caso, los resultados del análisis sobre la evolución de la investigación en Teoría de la Educación permiten matizar la queja que hemos visto formular desde el nivel metateórico, en el sentido de que lo que se ha perdido no es tanto la orientación práctica, como la identificación de esta orientación con la perspectiva tecnológica. De nuevo, estamos ante una evolución que es en cierto sentido lógica. Pensar la práctica educativa en toda su complejidad (Vázquez, 1989; Colom, 2002; Asensio, 2004) implica asumir que el irrenunciable criterio identificador de la Teoría-de-la-educación-comoteoría-para-mejorar-la-práctica debe considerarse, coherentemente, de un modo amplio, de tal manera que, para poder responder a esa complejidad y dinamismo imprevisible, se acepten diversos niveles de orientación a la práctica. Dicho de otro modo, pensar la educación en toda su complejidad implica aprender a proyectar un interés pedagógico, amplio y general, por saber desentrañar las múltiples vías de acceso a la realidad educativa, por 
adoptar amplios criterios de racionalidad, por aceptar una pluralidad de niveles de fundamentación teórica y por acoger orientaciones para la acción también en diferentes niveles $\left({ }^{25}\right)$. Como señalaba Puig Rovira en 1986, en nuestro campo

se entremezclan conocimientos e interpretaciones descriptivas sobre lo que es la educación, con saberes concernientes a cómo intervenir para que la acción educativa sea eficaz. Hablamos de dos momentos de reflexión porque ambos requieren de un esfuerzo de pensamiento racional. Sin embargo, en un caso su objetivo es predominantemente cognoscitivo, mientras que en el otro cualquier reflexión está guiada y tiende a solventar problemas prácticos, planteados durante la labor educativa (...) Ambos tipos de saber pueden relacionarse, pero no tienen por qué hacerlo necesariamente siempre así: podemos tener explicaciones del hecho educativo que, al menos de ?nomento, no nos ayuden a mejorar la intervención formativa. Aunque también podemos tener otras que sí la mejoran. Por otra parte, se conocen modos eficaces de intervención educativa no explicados mediante teorías, pero sin duda útiles (Puig Rovira, 1986, p. 23, cursiva añadida).

(25) los corolarios de esta tesis son varios, algunos vamos a analizarlos, otros quedan sólo apuntados: 1) hay que evitar que la visión integradora y abierta de nuestra disciplina se termine identificando con una claudicación del empeño tecnológico de la Teoría de la Educación; 2) la tarea constitutivamente central de la Teoría de la Educación debe seguir siendo la mejora de la práctica educativa, esto es, de las condiciones estructurales y concatenadas de las acciones;

3) atender a la práctica desde una Teoría de la Educación abierta e integradora no significa que, en todos los casos, la eficacia de esa práctica se resuelva en términos de tecnologías pedagógicas generales y específicas; 4) para lograr una Teoría de la Educación realmente abierta, integradora y sintetizadora, es necesario delimitar y diferenciar más de lo que se ha hecho hasta ahora, la explicación y comprensión propia de la teoría, de los conocimientos tecnológicos de transformación; 5) resulta gremialmente absurdo mantenerse en el "café para todos» sin reparar que los cultivadores de la Teoría de la Educación no son clones de un solo paradigma, sino investigadores que, en respuesta a la complejidad del mundo educativo, terminan por elaborar un amplísimo abanico de tendencias que van desde las más teóricas a las más prácticas. 
La Teoría de la Educación debe mantenerse siempre en una perspectiva abierta, integradora y sintetizadora porque hay espacios de reflexión y actuación, así como retos de conocimiento o preocupación en las líneas más vanguardistas de las ciencias sociales, que no dejan otra alternativa si es que queremos participar de todas las posibles preocupaciones formativas del hombre. Pero, al mismo tiempo, debemos aprender a compaginar este escenario gnoseológico con la pretensión de restituir lo pedagógico en el centro del discurso, eso sí lo pedagógico en su extensión total, por decirlo con las mismas palabras de Walsh.

Ya no podemos — ni debemos — restituir lo pedagógico centrándonos en las posibilidades de una única perspectiva práctica ni de un único discurso para la Teoría de la Educación. El empeño en erigir una única vía de análisis que valide como relevante 0 científico al discurso de la Teoría de la Educación, no sólo iría en contra de las tendencias actuales en los más variados campos de conocimiento, sino que nos haría perder la capacidad de enfrentarnos a la complejidad de los retos y problemas educativos. Además, considerar lo pedagógico y lo educativo en su extensión total implica asumir no sólo una pluralidad de enfoques prácticos, de metodologías de acción, de formas de transformar la realidad educativa sino, también y, en la circunstancia actual cabría decir muy especialmente, la necesidad de recuperar y, en su caso, establecer argumentos teóricos explicativos que justifiquen la validez o el sentido educativo de nuestras propuestas.

Para nosotros, el problema de la Teoría de la Educación no es, como algunos tienden a señalar, su excesiva especulación. Muchos pagaríamos hoy lo que fuera por poder disfrutar de un buen estudio teórico-especulativo acerca de la educación. Lo que nos debería irritar, y con razón, son estudios teóricos en los que lo pedagógico o lo educativo no constituye el centro del discurso 0, peor, es intercambiable por otros intereses y, en muchos casos, reducible a otras palabras: social, cultural, moral, etc. El problema de la Teoría de la Educación tampoco es, como otros tienden a indicar, su afán tecnológico, analítico y sus aspiraciones a establecer conexiones cuasi- 
nomológicas, lineales y anticipatorias de efectos. También pagaríamos una buena suma por este tipo de estudios. Quizás los mejores frutos pedagógicos de la Teoría de la Educación en nuestro país, en los últimos años, los encontramos, precisamente, en trabajos analíticos, muy concretos, definidos y centrados en temas bien delimitados 0 , y esto es más importante, que se delimitan artificialmente, sí, como hace cualquier investigador, pero para conocer mejor su funcionalidad pedagógica y, no precisamente, por lo menos en algunos casos, para desatender la complejidad inherente a las situaciones educativas. Nos referimos con esto a textos teóricos sobre educación moral, intercultural, inmigrantes, ambiental, no formal, currículo, cívica, nuevas tecnologías, formación del profesorado, etc. Dicho esto, debemos reconocer que algunas de las críticas que se han hecho a un enfoque tecnológico de la práctica educativa, tienen razón cuando consideran que se ha quedado, en muchos casos, en una metateoría tecnológica. Y lo mismo podría plantearse con respecto a una mera metateoría ética, deliberativa 0 sociocrítica. Para lograr constituirnos en un saber realmente abierto y sintetizador de lo que se piensa e investiga en todos los saberes científicos y humanísticos, y de lo que se «mueve» dentro de las dinámicas culturales y sociales, no podemos cuestionar ni renunciar a ningún nivel de especulación, ni de análisis artificialmente simplificados. Lo que tenemos que lograr es saber volver a los problemas más urgentes del mundo educativo, para ofrecer una mediación, de carácter pedagógico, que ayude a orientar la práctica educativa.

La Teoría de la Educación es todavía joven en nuestro país. Tal vez lo que ciertos autores valoran como avances 0 retrocesos, expectativas no cumplidas, enfoques excesivamente especulativos, etc., no sea más que la expresión dubitativa de un saber que trata de avanzar en el conocimiento de la educación procurando abarcar toda la complejidad de ésta. Si esto, en alguna medida, es cierto, consideramos que vamos por un buen camino, porque no podremos hablar de rigor en el saber pedagógico hasta que no logremos desentrañar las múltiples vías de acceso a lo educativo: agentes, políticas, fines, contextos, escuelas, familias, etc. Como cultivadores de la 
Teoría de la Educación, lo que sería motivo de preocupación es que ese crecimiento de estudios y análisis, especulativos o no, tan variado y rico, no lograse ir integrándose en un conocimiento sintético de la educación. Tal vez sólo estemos, como siempre, ante otro momento de transición, ahora centrado en múltiples contextos y discursos, y a la espera de que mañana también logremos sintetizar las condiciones básicas estructurales de los procesos educativos que, con toda seguridad, laten bajo las múltiples expresiones de lo educativo. Todos sabemos que cada vez es más difícil encontrar entre las jóvenes generaciones de teóricos de la educación — las que ni han conocido la «lucha ascética» ni se identifican ya con los hijos de la postguerra - generalistas del proceso educativo. El temor postmoderno a las grandes construcciones teóricas, ha forzado a especializarse en áreas 0 temas muy específicos. En este trabajo henos tratado de argumentar que esto, en sí mismo, no tiene por qué suponer un déficit. El reconocimiento de la complejidad de la educación no admite los planteamientos unidireccionales. La disgregación de discursos se convierte en un problema cuando hace que nos olvidemos de la mirada estrictamente educativa, de lo educativo y pedagógico en su extensión total, ya sea desde la teoría más especulativa 0 desde la práctica más concreta. 
Asensio, José M. (2004). Una educación para el diálogo. Barcelona, Paidós.

Bárcena, F.; Gil, F. y Jover, G. (1991). La dimensión ética de la actividad educativa. Notas críticas para el replanteamiento de un problema. Bordón, (43), pp. 259-269

Bárcena, F.; Gil, F. y Jover, G. (1993). The Ethical Dimension of Teaching: A Review and a Proposal, The Journal of Moral Education, (22), pp. 241-252.

Borrell, N.; Benedito, A. y Millan, M.D. (1979). La secció de pedagogía de la Universität de Barcelona. Barcelona: Universität de Barcelona, Institut de Ciències de l'Educacio.

Campillo, J. (1974). Teoría de la Educación. En A. Millán Puelles (Dir.), Teoría de la Educación (Filosofía de la Educación) (vol.1, pp. 17-24). Madrid: UNED.

Capitán. A. (1977). Teoría de la Educación. Granada: ICE.

Colom, A. J. (1992). El saber de la Teoría de la Educación. Su ubicación conceptual. Teoría de la Educación. Revista Interuniversitária, (4), pp. 11-19.

Colom, A. J. (1997). La Teoría de la Educación: contexto actual de los estudios pedagógicos. En A. J. Colom (Coord.), Teorías e instituciones contemporáneas de la educacion (pp. 145155). Barcelona: Ariel.

Colom, A. J. (2002). La (de)construcción del conocimiento pedagógico. Barcelona: Paidós.

Colom, A. J. (2003). Lo político y lo comunitario en la teoría de la educación española: una revisión. En P. Ortega (Ed.), Teoría de la Educación, ayer y hoy (pp. 109-158). Murcia: Selegráfica.

Colom, A. J. y Rincón, J.C. (2004). Epistemología neoidealista y fracaso fundacional del saber educativo. Teoría de la Educación, Revista Interuniversitária, (16), pp. 19-47.

Dearden, R. F. (1984). Theory and practice in education. London: Routledge and Kegal Paul.

Escámez, J. (1988). La teoría pedagógica y el proceso educativo. En Sociedad Española de Pedagogía (Ed.), IX Congreso Nacional de Pedagogía. La calidad de los centros educativos (pp. 11-24). Alicante: Instituto de Estudios Juan Gil-Albert, Caja de Ahorros Provincial de Alicante.

Escámez, J. (2003). Los valores y la educación en España. En P. Ortega (Ed.), Teoría de la Educación, ayer y hoy (pp. 205-237). Murcia: Selegráfica.

Escolano, A. (2002). La educación en la España contemporánea. Políitcas educativas, escolarización y culturas pedagógicas. Madrid: Biblioteca Nueva.

Fermoso, P. (1976). Teoría de la Educación. Madrid: Agulló.

Foucault, M. (1991). Saber y verdad. Madrid: La Piqueta.

García Carrasco, J. y García Del Dujo, A. (2001). Teoría de la educación 11. Procesos primarios de formación del pensamiento y la acción. Salamanca: Ediciones Univer sidad de Salamanca.

Geertz, C. (1994) Conocimiento local. Barcelona: Paidós. 
Gil, F. (1997). Tecnología educativa, en AAVV., Filosofía de la Educación hoy. Diccionario (pp. 538-541). Madrid: Dykinson,

Gil, F. (2001). Educación y crisis del sujeto. Teoría de la Educación, Revista Interuniversitária, (13), pp. 45-68.

Gil, F. (2003). La relevancia práctica de la Filosofía de la Educación. En M. García Amilburu (Ed.), Claves de la Filosofía de la Educación (pp. 187-208). Madrid: Dykinson.

Hardie, C.D. (1968). Truth and Fallacy in Educational Theory. New York: Teachers College Press.

Hirst, P.H. (1983). Educational theory. En P.H. Hirst (Ed.), Educational theory and its foundation disciplines (pp. 3-29). London: Routledge and Kegan Paul.

Hirst, P.H. (1998). Philosophy of Education: The evolution of a Discipline. En G. Haydon (Ed.), 50 Years of Philosophy of Education (pp. 1-22). London: University of London, Institute of Education.

Ibáñez-Martín, J. A. (1989). El papel de la teoría de la educación en la formación del profesorado. En J. M. Esteve (Ed.), Objetivos y contenidos de la educación para los años noventa (pp. 143-148). Málaga: Universidad de Málaga.

Jover, G. (2001). Philosophy of Education in Spain at the Threshold of the 21st Century. Origins, Political Contexts, and Prospects, Studies in Philosophy and Education, (20), pp. 361-385.

Jover, G. (2002). Proyecto docente. Cátedra de Teoría de la Educación. Madrid: Universidad Complutense.

Jover, G. (2003). El aspecto ético en la configuración profesional de la educación. En M. Ruiz Corbella (Ed.), Educación moral: aprender a ser, aprender a convivir (pp. 169-188). Barcelona: Ariel.

Marín Ibáñez, R.(1983). Medio siglo de Pedagogía General, Revista Española de Pedagogía, (61), pp. 9-23.

Martínez Martín, M. y Buxarrais, M. R. (1992). La investigación en Teoría de la Educación, Teoría de la Educación. Revista Interuniversitária, (4), pp. 21-39.

Moore,T.W. (1983, 2a ed.). Introducción a la Teoría de la Educación. Madrid: Alianza.

0'Connor, D. J. (1967, 60 reimp.). Introduction to the Philosophy of Education. London: Routledge and Kegal Paul.

Ortega, P. (Ed.) (2003). Teoría de la Educación, ayer y hoy. Murcia: Selegráfica.

Puig Rovira, J. M.a (1986). Teoría de la educación. Una aproximación sistémico-cihemética. Barcelona: PPU.

Sarramona, J. (2000). Teoría de la educación. Reflexión y normativa pedagógica. Barcelona: Ariel.

Sarramona, J. (2003). La perspectiva tecnológica en la acción educativa. En P. Ortega (Ed.), Teoría de la educación, ayer y hoy (pp. 159-204). Murcia: Selegráfica.

Schriewer, J. (1996). Sistema mundial y redes de interrelación: La internacionalización de la educación y le papel de la investigación comparada. En M.A. Pereyra, et al., Globalización y descentralización de los sistemas educativos (pp. 17-58). Barcelona: Pomares-Cor redor.

Schriewer, J. (2000). Estudios multidisciplinares y reflexiones filosófico-hermenéuticas: la estructuración del discurso pedagógico en Francia y Alemania. En J. Ruiz Berrio (Ed), La cultura escolar de Europa. Tendencias históricas emergentes (pp. 231-269). Madrid: Biblioteca Nueva. 
Simon, B. (1999). Why No Pedagogy in England? En J. Leach y B. Moon (Eds.), Learners and Pedagogy (pp. 34-45). London: Paul Chapman.

Universidad de Madrid (1932). Facultad de Filosofía y Letras. Año académico de 1932-1933Madrid: Imp. de Galo Sáez.

Universidad de Madrid (1935). Facultad de Filosofía y Letras. Año académico de 1935-1936. Madrid: Imp. de Galo Sáez.

Vázquez, G. (1989). Hacia la Pedagogía como 'ciencia abierta'. En B. Delgado y M.L. Rodríguez (Eds.), Homenaje al profesor A. Sanvisens (pp. 243-255). Barcelona: PPU.

Walsh, P. (1993). Education and Meaning: Philosophy in Practice. London: Cassell. 